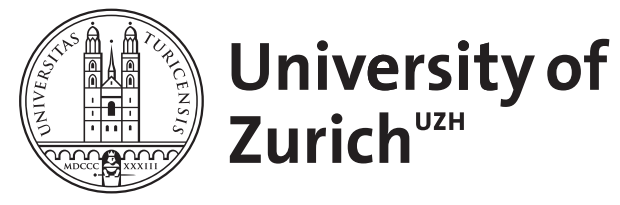

Zurich Open Repository and Archive

University of Zurich

University Library

Strickhofstrasse 39

CH-8057 Zurich

www.zora.uzh.ch

Year: 2014

Schwache Erzähler, starke Leser: Zum erzählerischen Programm im Vorwort von Gellius' Noctes Atticae

Beer, Beate

DOI: https://doi.org/10.1515/anab-2014-0106

Posted at the Zurich Open Repository and Archive, University of Zurich ZORA URL: https://doi.org/10.5167/uzh-104051

Journal Article

Published Version

Originally published at:

Beer, Beate (2014). Schwache Erzähler, starke Leser: Zum erzählerischen Programm im Vorwort von Gellius' Noctes Atticae. Antike und Abendland, 60:51-69.

DOI: https://doi.org/10.1515/anab-2014-0106 


\section{Schwache Erzähler, starke Leser: Zum erzählerischen Programm im Vorwort von Gellius’ Noctes Atticae}

In Gell. 3,19 werden dem Leser im erzählerischen Rahmen eines Gastmahls zwei Etymologien von parcus präsentiert. Beide Etymologien werden in Form von Zitaten aufgeführt und einander kontrovers gegenüber gestellt: Nachdem aus einem Buch des Grammatikers Gavius Bassus die Ausführungen zu parcus vorgelesen wurden, hält Favorinus seine eigene Theorie dagegen. Trotz dieser erzählerisch vielversprechenden Struktur - anlässlich eines Gastmahles kommt es zu einer kontrovers geführten Debatte - verblasst der Ich-Erzähler im Verlaufe des Kapitels vollständig. Was als Ich-Erzählung und Erlebnisbericht über ein Gastmahl begann, endet als lateinische Grammatik. Ja, man vermisst sogar die präskriptive Haltung eines grammatischen Textes. Denn das Ende des zweiten Zitats fällt mit dem Ende des Kapitels zusammen, und der Leser wird ohne abschliessenden Erzählerkommentar entlassen.

\section{Der aktive Leser als Konstrukt einer narrativen Strategie}

Der Erzähler der Noctes Atticae verzichtet weitgehend darauf, das Erzählte zu kommentieren und zu bewerten. Dieser vordergründige Verzicht auf einen erklärenden und konstruierenden Erzähler wird gemeinhin auch als Verzicht auf eine literarische Gestaltung gewertet. ${ }^{1}$ Im Folgenden soll diese Beobachtung zum Erzähler als narrative Strategie in den Noctes Atticae und als Mittel der Leserinvolvierung ${ }^{2}$ beschrieben werden. Auch ein Verzicht ist aus dem Blickwinkel der Pragmatik - eine, hier narratologische, Aussage. In einem weiteren Schritt wird es im zweiten Teil des Aufsatzes darum gehen, den kulturellen Kontext der Leserinvolvierung, in der Art wie sie in den Noctes Atticae ausgestaltet ist, abzustecken. Gellius' literarisches Schaffen fällt in die Zeit der zweiten Sophistik, und so scheint es naheliegend, den Text eines Autors, der in so hohem Masse rezipiert wie Gellius, auf Einflüsse dieser zeitgenössischen kulturellen Bewegung zu untersuchen. Durch diese Kontextualisierung, so wird zu zeigen sein, lassen sich neben der Leserinvolvierung weitere Einzelbeobachtungen, die sich insbesondere aus der vergleichenden Gegenüberstellung zu Plinius ergeben, der mit der naturalis historia als ein Vorgänger für Gellius’ Miszellanschrift gelten kann, zu einem Konzept von Literarizität zusammenfügen, das der zweiten Sophistik zuzuschreiben ist. Da dieser Aufsatz am Anfang eines weiter gefassten Projekts zur Narrato-

${ }^{1}$ Unter anderen vgl. Ameling 1984, 484: «Die «Noctes Atticae` des Aulus Gellius sind literarisch wenig bedeutend, doch ihre Nützlichkeit ist unbestritten.» Zur moderneren Einschätzung vgl. Beall 1999, 55: $\ll \ldots$, and he now receives credit for a certain originality and creativity.»

2 Der Ausdruck ‘Leserinvolvierung» steht in Anlehnung an Pausch 2011, 191-250: «V. Der involvierte Leser: Spannung als historiographische Strategie». 
logie in den Noctes Atticae steht, wird sich die vorliegende Untersuchung auf das Vorwort ${ }^{3}$ beschränken.

Es ist bekanntermaßen das Vorwort, das dem Leser verspricht, etwas mehr vom erzählerischen Programm eines Textes zu erfahren. In den Noctes Atticae entfaltet die Praefatio weite intertextuelle Bezüge, so dass sie vom literarischen Diskurs, in dem sich der Erzähler positioniert, sowie von seinem literarischen Gestaltungswillen zeugt. Ihre Sonderstellung innerhalb der Noctes Atticae ist dadurch hervorgehoben, dass nach ihr nicht unmittelbar die Reihe der einzelnen Kapitel, sondern zunächst eine Inhaltsübersicht folgt. Die Praefatio ist damit der Aufgabe der konkreten Inhaltsangabe enthoben und kann sich umso stärker dem Entwurf des impliziten Lesers ${ }^{4}$ widmen. Gerade der Leser, der aktiviert sein soll, ist auf eine gewisse Hilfestellung zur Aktivität angewiesen. Er wird motivierter an seine Lektüreaufgabe herangehen, wenn er weiss, welchen Nutzen oder welche Unterhaltung er daraus ziehen kann.

Die intertextuellen Bezüge, die in der Praefatio der Noctes Atticae entworfen werden, fanden in der Forschungsliteratur schon mehrfach Behandlung. An erster Stelle sind die Arbeiten von Minarini 2000 und Holford-Strevens $2003 \mathrm{zu}$ nennen, die beide das enge und ambivalente Verhältnis zum Vorwort von Plinius, nat. erläutern. In Ergänzung zu diesen beiden Arbeiten soll in der folgenden Darstellung der Vergleich mit Plinius dazu dienen, die Positionierung im literarischen Diskurs, die der Erzähler der Noctes Atticae im Vorwort selbst vornimmt, zu erhellen. Die Auseinandersetzung mit Plinius ist für den Erzähler der Noctes Atticae nicht nur Ausdruck eines persönlichen Konkurrenzverhältnisses zwischen zwei römischen Miszellanautoren, sondern umfasst auch deren literarischen Hintergrund. Einen weiteren Bogen schlägt Ker 2004, der Gellius’ Praefatio für seine Beschreibung der lucubratio als eines literarischen Topos heranzieht. Vardi 1993 und Korenjak 1998 bieten eine Analyse des Titels. Dabei geht Vardi von der Funktion des Titels und Korenjak vom Aristophanes-Zitat in Praefatio 21 aus. Riggsby 2007 kontextualisiert die Praefatio aufgrund des Verweises auf ein Inhaltsverzeichnis und ergründet die Entstehung dieses Hilfsmittels. Die vorliegende Untersuchung wird besonders die bei Minarini, Holford-Strevens, Vardi und Korenjak gebotenen intertextuellen Analysen nutzen, um die Praefatio aus narratologischer Perspektive zu beleuchten. Dazu sollen zunächst in der Reihenfolge ihres Auftretens in der Praefatio die Aussagen erörtert werden, die der Konstruktion des impliziten Lesers dienen.

Zwar sind die ersten Sätze des Vorworts verloren, doch dürfte sich der Umfang des verlorenen Textes in Grenzen halten, da die Überlieferung noch mit der Widmung einsteigt, die wir am Anfang eines Textes erwarten, wenn denn eine solche vorhanden ist. Unser Erzähler widmet den Text in Praefatio 1 seinen Kindern: liberis ... meis. Sie sollen sich dereinst bei der Lektüre von ihren Pflichten erholen können. Gellius’ Miszellanschrift ist damit ein Mittel zur Entspannung in der Freizeit, und zwar, da vom Vater verordnet, ein nach römischer Tradition adäquates. In zahlreichen Kapiteln wird der Erzähler ferner am eigenen Beispiel illustrieren, was als sinnvolle Freizeitgestaltung zu betrachten ist. ${ }^{5}$

\footnotetext{
${ }^{3}$ Im Folgenden: Praefatio.

${ }^{4}$ Der Begriff 'Leser bezeichnet in dieser Arbeit, sofern nicht anders angegeben, den impliziten Leser nach Iser 1976, 37-67. Der Begriff der Erzählstrategie umfasst im Folgenden die narratologischen Mittel, die auf den impliziten Leser zielen und ihn zu aktiver Lektüre, d.h. zu Sinnstiftung durch eigene Konstruktion und Ergänzung der Darlegungen, auffordern.

${ }^{5}$ Dass eine sinnvolle Freizeitbeschäftigung etwa darin besteht, gegenüber Freunden in ungezwungenem Ton seine Bildung zu belegen, zeigt bspw. Gell. 1,2.
} 
Welcher Art die angestrebte Entspannung ist, kann der Leser aber bereits dem zweiten Satz, Praefatio 2, entnehmen. Denn das Mittel zu angemessener Entspannung ist aus ebendieser heraus entstanden:

Usi autem sumus ordine rerum fortuito, quem antea in excerpendo feceramus. Nam proinde ut librum quemque in manus ceperam seu Graecum seu Latinum vel quid memoratu dignum audieram, ita quae libitum erat, cuius generis cumque erant, indistincte atque promisce annotabam eaque mihi ad subsidium memoriae quasi quoddam litterarum penus recondebam.

Wir sind ferner ${ }^{6}$ nach einer zufälligen Anordnung der Dinge verfahren, die wir zuvor beim Exzerpieren vorgenommen hatten. Denn ebenso wie ich ein jedes Buch in die Hand bekommen hatte, sei es ein griechisches oder ein lateinisches, oder etwas Erinnerungswürdiges gehört hatte, habe ich es, so wie es beliebte und welcher Art es war, unterschiedslos und vermischt festgehalten und mir zur Gedächtnisstütze gewissermassen als Wissensvorrat gespeichert. ${ }^{7}$

Der Erzähler, in erster Linie selbst ein Leser, beschreibt seine eigenen Lektüregewohnheiten. Aus diesen ergibt sich, dass die Sammlung thematisch offen und ungeordnet ist: indistincte atque promisce. Sein gestalterisches Motto ist also die bunte Durchmischung, die disparilitas, wie er selbst in Praefatio 3 erklärt. Diese Durchmischung verschiedener Formen und Themen wird präzisiert durch die Metapher des Wissensvorrats, litterarum penus, die auch die Motivation für die Sammlung impliziert. Das Wort penus wird im Kapitel 4,1 ausführlich erläutert, ${ }^{8}$ und so scheint es auf der Suche nach dem erzählerischen Programm geboten, die dort gegebene Definition für das Verständnis der Metapher in Praefatio 2 heranzuziehen. Ein intratextueller Bezug auf die Praefatio kann nicht zuletzt auch die prominente Platzierung der Definition im Eröffnungskapitel zum vierten Buch begründen.

Gewährsmann für die Definition in 4,1 ist Favorinus, dem zahlreiche weitere Eröffnungskapitel gewidmet sind. ${ }^{9}$ Der Begriff penus und seine Erörterung werden in 4,1,1-2 vom Erzähler mit einem Augenzwinkern eingeführt. Denn der erzählerische Rahmen zur sachlichen Ausführung schildert, wie Favorinus und andere gebildete Männer der Oberschicht im Vorhof des Palastes auf eine Audienz warten. Die Gruppe der Wartenden wird durch das Thema ihres Smalltalks als zur Oberschicht gehörig charakterisiert. Denn man diskutiert über dies und jenes aus dem Bereich der Philologie. ${ }^{10}$ Dann fokussiert der Erzähler auf einen Grammatiker. Indem dieser Grammatiker aber namenlos bleibt (quispiam grammaticae rei ditior) und seine Gesprächsbeiträge als Kleinkram aus der Dorfschule (scholica quaedam nugalia) bezeichnet werden, stigmatisiert der Erzähler ihn gleich von Be-

\footnotetext{
${ }^{6}$ Die Partikel autem ist hier wohl nicht adversativ, sondern amplifizierend, und damit nach dem für Gellius typischen Stilmittel der Amplificatio aufzufassen. Marache 1981 führt in seiner Untersuchung des Stils in Praefatio als besonderes Charakteristikum die Bildung von Wortpaaren an. Die Wortpaare bestehen aus Synonymen oder aus Wörtern mit zumindest ähnlicher Bedeutung. Im ersten Fall verleihen sie dem geforderten Ausdruck mehr Gewicht. Bei Paaren ähnlicher Bedeutung entsteht ein Bedeutungskonglomerat, dessen Bestandteile sich gegenseitig präzisieren. Als erstes Beispiel in Gell. steht laxari indulgerique in Praefatio 1. Maraches Beobachtungen können aber auch über die Praefatio hinaus für die einzelnen Kapitel gelten.

7 Die Übersetzungen stammen von der Verfasserin.

8 So zuerst Faider 1927, 201 und Cavazza 1985, 354.

9 Nach Beall 1988, 195: 2,1; 3,1; 4,1; 12,1; 14,1; 18,1; 20,1.

10 Zur Bedeutung von Sprache und Literatur für die Bildung der Oberschicht vgl. Schmitz 1997.
} 
ginn an als Spottobjekt. Die gewichtige Haltung dieses Grammatikers, der mit erhobenen Augenbrauen (cum arduis superciliis), gesetztem Tonfall (vocisque et vultus gravitate) und mit der Miene eines Deuters der Sibyllinischen Bücher (tamquam interpres et arbiter Sibyllae oraculorum) zu seiner Rede ansetzt, lassen in ihrer Übertreibung nach dem Muster eines Hypsos-Bathos-Wechsels eine wahre Lächerlichkeit erwarten. Nachdem der Erzähler die Nennung des Wortes, das durch den Grammatiker erörtert werden wird, durch die lange Redeeinleitung weiter herausgezögert hat, wird dem Leser endlich penus als Begriff und sachliches Thema des Kapitels entdeckt: Tum aspiciens ad Favorinum, quamquam ei nondum etiam satis notus esset: “"penus» quoque〉 inquit ‘...). Diese erzählerische Strukturierung der Rahmenhandlung und Einleitung zur Erörterung von penus zielt auf eine effektvolle erste Nennung des sachlichen Themas. Der Spott zielt dabei nicht auf den Begriff selbst, sondern auf die Art des Interesses, das der Grammatiker für penus bekundet. Ihn beschäftigen nämlich die verschiedenen Formen, in denen das Wort auftritt, während Favorinus die Diskussion sogleich auf die Semantik und damit indirekt auch auf den metaphorischen Gebrauch in der Praefatio lenkt.

Der Begriff bezeichnet Favorinus zufolge einen Vorrat, der behütet im Inneren des Hauses lagert und der so viele verschiedene Dinge umfasst, dass man ihn anhand einer Liste nicht fassen kann. Wie die Noctes Atticae zeichnet sich dieser Vorrat also durch seine disparilitas aus. Er ist ausserdem sehr reichlich im Umfang. Und ebenso wie die Noctes Atticae, so kann der Leser aus dem Umfang folgern, ist er die Frucht ausdauernden Zusammentragens. ${ }^{11}$ Gerade der Umstand, dass er ferner gemäss Gell. 4,1,17 langfristig und nicht für den aktuellen Bedarf angelegt ist, charakterisiert ihn: Nam quae ad edendum bibendumque in dies singulos prandii aut cenae causa parantur, ‘penus> non sunt. Auch dies untermauert unter Rückbezug auf die Metapher in Praefatio 2 das literarische Programm in den Noctes Atticae als Freizeitaktivität. Die Lektüre der Miszellanschrift gehört nicht zum Tagesgeschäft des Lesers aus der römischen Oberschicht. Ebenso wenig bringt sie ihm unmittelbaren Nutzen in seinen Aufgaben politischer und juristischer Amtsführung. Die Lektüre des Wissensvorrats, auf den er sporadisch zurückgreifen kann, vermittelt vielmehr Bildung und Lebenshaltung und dient mithin der Charakterformung. So erstaunt es auch nicht, dass der mit penus bezeichnete Vorrat für den Hausherrn und für dessen Kinder zum Gebrauch bestimmt ist:

Penus est ..., quod ipsius patrisfamilias 〈aut matrisfamilias〉 aut liberum patrisfamilias $\langle$ aut familiae eius, quae circum eos aut liberos eius est et opus non facit, causa paratum est.

Der Vorrat ist das ..., was für den Familienvater 〈oder für die Hausherrin〉 oder für die Kinder des Familienvaters 〈oder für das Gesinde〉 desselben, das um diese (beiden) oder um seine Kinder herum sich aufhält und keine Arbeit zu erledigen hat, angelegt ist.

Die Nutzniessenden des penus sind zugleich die Leser von Noctes Atticae. Die in 4,1 gegebene nachträgliche Definition des Wortes, das in der Praefatio zur Beschreibung des Textes herangezogen wurde, strukturiert den Text, indem sie verdeckte Sinnbezüge konstruiert, die implizit bleiben. Die Textstruktur stellt den Leser vor die Aufgabe, die Textfragmente, die sich inhaltlich ergänzen, auch wenn sie strukturell voneinander getrennt stehen, zusam-

11 Gell. 4,1,15: Meministi enim, credo, quaeri solitum, quid Vergilius dixerit, "penum struere» vel «longam» vel "longo ordine». 
menzuführen. Ebenso wird der Hausherr je nach Bedarf die Gegenstände aus dem Vorrat heraussuchen und für den konkreten Verwendungszweck zusammenstellen, die im Vorrat selbst an unterschiedlichen Stellen lagern. Dieses narratologische Verfahren kann als Leserinvolvierung verstanden werden. Es ist Ausdruck der in der Textstruktur angelegten Leserrolle.

In der Praefatio wird als nächstes die Titelwahl erläutert. Der Erzähler gibt sich bescheiden. Er habe, so heisst es in Praefatio 4, nicht den Vergleich mit ausgefalleneren Titeln gesucht und nicht versucht, die Feinheit anderer Titel nachzuahmen: nibil imitati festivitates inscriptionum, quas plerique alii utriusque linguae scriptores in id genus libris fecerunt. Es werden Beispiele für ausgefallenere und, bunt gemischt, weniger ausgefallene Titel genannt, darun-

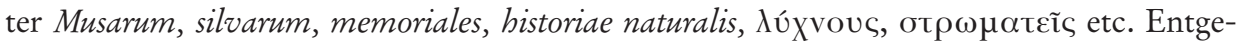
gen der bescheidenen Einleitung des Erzählers zeigt sich, dass sich der Titel Noctes Atticae gut in diese Beispiele einreiht und sicher nicht zu den langweiligsten gehört. ${ }^{12}$ Der Leser wird nämlich, so wie wir das gerade getan haben und wie das in der Gellius-Forschung überhaupt bis heute geschieht, ${ }^{13}$ den Titel nach dieser bescheidenen Einführung nur umso aufmerksamer mit den aufgezählten Beispielen vergleichen. Die Bescheidenheitsgeste gründet auf einer Floskel, die in ihrer Wirkung wohl am ehesten mit der Praeteritio verglichen werden kann. In einer Praeteritio formuliert ist der Titel so gewöhnlich, dass man ihm weiter keine Beachtung zu schenken braucht. Gerade durch diesen indirekt deiktischen Gestus des Erzählers wird der Leser animiert, den Wahrheitsgehalt der Aussage zu überprüfen, zumal ihm die Daten dazu so aufdringlich präsentiert werden. Die Erklärungen zum Titel stehen damit im Dienste der narrativen Erzählstrategie in den Noctes Atticae und ihrem Ziel der Leserinvolvierung. Die Einführung des Titels geht in der Leserinvolvierung aber noch einen Schritt weiter. Denn die zum Vergleich genannten Titel werden in einer offenen Liste aufgeführt, die dem Leser nicht nur eine Information oder ein Wissen vermittelt - eben über andere Titel anderer Miszellen und Miszellanschriften -, als vielmehr auch weitere Fragen aufwirft. So wird der Leser zum einen aktiviert, selbst nach Titeln aus seiner Lektüreerfahrung zu suchen, welche die Liste und somit die Notizen des Erzählers ergänzen könnten. Zum anderen dürfte der Leser sogleich die Autoren zu erraten versuchen, auf die mit dem genannten Titel ja jeweils indirekt verwiesen wird. Der Erzähler gibt seinem Leser nur die halbe Information, wodurch dieser zur Ergänzung aufgefordert ist. ${ }^{14}$

Entsprechend der expliziten Zurückhaltung in seiner literarischen Selbstdarstellung gibt der Erzähler seinem Titel eine ausserliterarische Motivation. Zeit und Ort der Niederschrift haben, so der Erzähler, in den Titel Eingang gefunden: ex ipso loco ac tempore hibernarum vigilarum Atticas noctes inscripsimus. Vardi 1993 hält fest, dass der Titel seine Funktion, nämlich dem Leser Anhaltspunkte zum Inhalt zu geben, nicht erfülle. Der Leser steht also bereits beim Titel vor der Aufgabe aktiver Sinnstiftung. Der Umstand, dass zahlreiche Rahmenhandlungen den Ich-Erzähler aber nicht in Athen, sondern in Rom oder in einer anderen Stadt Italiens zeigen, deckt den Symbolcharakter des Titels auf. Athen galt nicht

12 Holford-Strevens 2003, 27, spricht dieser Titelauswahl sogar eine «increasing dullness» zu, die der Erzähler durch seine zur Schau gestellte Bescheidenheit entlarvt und der Lächerlichkeit preisgibt. Minarini 2000, 536, hält fest, dass die Bescheidenheit nur eine scheinbare ist.

${ }^{13}$ Vgl. die Arbeiten von Vardi 1993, Minarini 2000, Holford-Strevens 2003.

${ }^{14}$ Diese Leserreaktion dokumentiert Minarini 2000, 536: «... per le quali si è cercato di individuare i rispettivi autori, che vanno da scrittori arcaici, come Accio, ad altri contemporanei a Gellio, come Favorino.» Vgl. Faider 1927, 203-209 und Cavazza 1985, 347-352. 
erst seit dem 2. Jh. n. Chr. als intellektuelles und kulturelles Zentrum der Oberschicht. Dem gebildeten Leser, der selbst seine nächtlichen Mussestunden der Bildung widmete (eben auch durch die Lektüre der Noctes Atticae), dürfte sich die symbolische Bedeutung des Titels problemlos erschlossen haben. Der Titel ist damit ein Mittel zur Zuordnung und Ausgrenzung innerhalb der Leserschaft, durch welches der Erzähler sein Bild vom Leser entwirft.

Die Textsammlung, so wird in Praefatio $13 \mathrm{f}$. bestätigt, ist in der Nacht, bei Kerzenschein und als Frucht der lucubratio entstanden. Sie bietet keine tiefgehenden, abschliessenden Untersuchungen, sondern vielmehr Kostproben, die zum weiteren Studium ermuntern sollen:

Non enim fecimus altos nimis et obscuros in his rebus quaestionum sinus, sed primitias quasdam et quasi libamenta ingenuarum artium dedimus, quae virum civiliter eruditum neque audisse umquam neque attigisse, si non inutile, at quidem certe indecorum est. $\mathrm{Ab}$ his igitur, si cui forte nonnumquam tempus voluptasque erit lucubratiunculas istas cognoscere, petitum impetratumque volumus, ut in legendo, quae pridem scierint, non aspernentur quasi nota involgataque.

Wir haben nämlich in diesen Dingen keine allzu tiefgehenden und dunklen Fragestellungen angestellt, sondern gewisse Anfänge und gleichsam Kostproben der standesgemässen Wissenschaften gegeben, von denen niemals gehört zu haben und mit denen niemals in Berührung gekommen zu sein, für den gesellschaftlich gebildeten Mann wenn nicht schädlich, so doch unziemlich ist. Von ihnen (scil. den Lesern) also, wenn einer zufällig manchmal die Lust und die Zeit hat, diese Spielereien bei Kerzenschein kennenzulernen, wollen wir nachdrücklich verlangen, dass sie bei der Lektüre von dem, was sie schon längst gekannt haben, es nicht als gewöhnlich und allgemein bekannt verachten.

Der Erzähler beschreibt sein Auswahlkriterium, nachdem er sich bereits in Praefatio 12 von Vielwisserei, die um ihrer selbst betrieben wird, durch ein Zitat Heraklits von Ephesos ab-

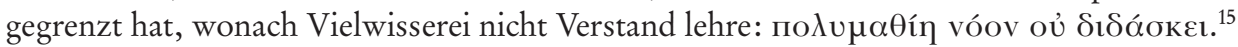
Als Auswahlkriterium diente ferner gemäss Praefatio 15 die Maxime, dass nichts, was in den Schulen heruntergebetet und in den Kommentaren breit getreten wird, in die Sammlung Eingang finden sollte. Mit der Kritik an Vielwisserei und der Ablehnung gegenüber einer Form von Wissen, das als schulmässig empfunden wird, zeigen sich Abgrenzungstendenzen des Erzählers gegenüber anderen Textsammlungen mit enzyklopädischem Charakter. Er lässt damit auch durchblicken, dass seine Praefatio 2 zufolge scheinbar wahllos zusammengetragene Miszellanschrift ${ }^{16}$ durchaus auf einer Auswahl und Konstruktion beruht.

Nachdem der Erzähler in dieser Weise das Feld seiner Interessen abgesteckt hat, nimmt er eine weitere Abgrenzung bezüglich seiner Rezipienten vor. Er beschreibt in Praefatio 19 seine Haltung gegenüber Kritikern, die sich nie die Zeit für wissenschaftliche Studien nehmen mögen und die nie diese Art von Nachtwachen wachten (nullas hoc genus vigilias vigilarunt). An dieser Stelle lässt der Erzähler ein weiteres Mal andere für sich sprechen. Er beruft sich für den Umgang mit Ungebildeten, die als solche als potentielle Kritiker gesehen werden, zum einen auf ein altes lateinisches Sprichwort (vetus adagium) und zum anderen auf Aristophanes, Ran. 354-356 und 369-371. Der Sprecher des Zitats aus den Fröschen spricht anstelle des Erzählers der Noctes Atticae. Das Zitat ist innerhalb der Noctes Atticae

15 Das Zitat ist aufgeführt als fr. 40 Diels Kranz, wo es als Testimonium in Athen. 13,610B ausgewiesen wird.

16 Praefatio 2: Nam proinde ut librum quemque in manus ceperam seu Graecum seu Latinum ... 
und als versetzte Erzählerrede für den Leser nur sinnvoll, wenn es gelingt, seine Aussage in den Zieltext, die Noctes Atticae, einzubinden und als Aussage über diesen zu deuten. Ähnlich wie in Gell. 3,19 oder 4,1 steht der Leser vor der Aufgabe, Analogien zwischen diskreten Texteinheiten zu konstruieren. Dem Leser erschliesst sich die Aussage über die Rezipienten der Noctes Atticae an dieser Stelle der Praefatio nur durch den Filter des Zitats. Es übernimmt die kognitive Funktion einer Metapher ${ }^{17}$, indem es die Noctes Atticae als eine Mysterienfeier beschreibt. Korenjak 1998 hält fest, dass das griechische Zitat als vox mystica fungiere, die nur von den Initiierten bzw. (ausserhalb der Metapher) nur von der Bildungsoberschicht verstanden werden kann. ${ }^{18}$ Im Zitat liegt ein Schlüssel zum Verständnis von Gellius' Miszellanschrift und durch die griechische Sprache und den intertextuellen Bezug eine Aktivierung des gebildeten Lesers. Im Zitat heisst es, dass Uneingeweihte der heiligen nächtlichen Feier fernbleiben mögen:

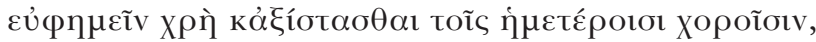

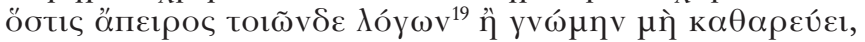

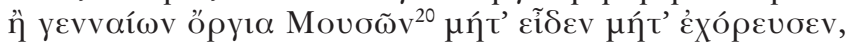

...

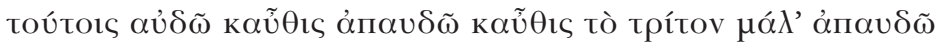

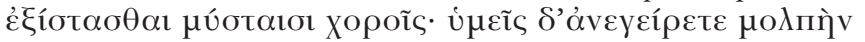

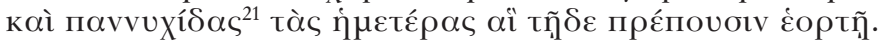

$\mathrm{Zu}$ schweigen und sich aus unseren Chören zurückzuziehen, das gehört sich für den, der in dieser Gesprächsführung unkundig ist oder keinen reinen Geist hat oder nie die Feiern der edlen Musen sah noch an ihnen tanzte. ... Diesen rufe ich wieder und wieder zu, und zum dritten Mal rufe ich ihnen wirklich zu, den Chören der Mysten fernzubleiben; ihr aber nehmt Gesang und Tanz wieder auf und unsere nächtlichen Feiern, die sich für dieses Fest geziemen.

Die Metapher strahlt über das Zitat hinaus in den Kontext aus. Denn bereits Praefatio 19 überhöht die Spielerei bei Kerzenschein zum Musenkult. Durch das Aristophanes-Zitat wendet sich der Sprecher dialogisch an einen Adressaten. In Ran. 354-356 und 369-371 liegen sogar zwei Adressatengruppen vor: die der Uneingeweihten (öotıৎ) und die der

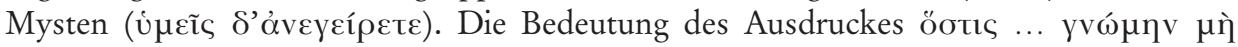
к $\alpha \theta \alpha \rho \varepsilon u ́ \varepsilon 1$ wird bestimmt durch die in Ran. 358-367 nachfolgende Beschreibung derer, die den Mysterien fern bleiben sollen. Sie werden durch ihr unmoralisches Verhalten charakterisiert. Entsprechend grosse Bedeutung kommt in den Noctes Atticae ethischen Fragestellungen zu, wie etwa das Heraklit-Zitat gegen Vielwisserei in Praefatio 12 verdeutlicht, ebenso wie die gegen die Mirabilientradition in Plinius, nat. gerichteten Kapitel Gell. 9,4 und 10,12 und weiter das Homer-Zitat in 14,6. Die Beschwörung des «reinen Geistes» hat

\footnotetext{
17 Black 1996, bes. 72.

18 So Korenjak 1998, 82. Unter Verweis auf die bei bei Aelius Aristides in Panath. 330 vorgenommene Parallelisierung von den eleusinischen Mysterien einerseits und von Bildung andererseits als zwei Errungenschaften und Gaben der Athener bestimmt Korenjak ferner die im Titel Noctes Atticae angelegten Implikationen auf die in Attika und des Nachts gefeierten eleusinischen Mysterien.

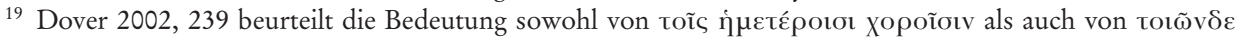
$\Lambda o ́ y \omega v$ als «completely ambivalent».

20 Auch in Ran. wird der Mysterienkontext metaphorisch gebraucht. Die zitierten Verse changieren zwischen Mysterium und Komödie.

${ }^{21}$ паvvvXís ist das griechische Pendant (ohne Diminutiv) zu lucubratiunculae in Praefatio 14.
} 
auch gruppenbildende Funktion. Auf unseren Zieltext, die Noctes Atticae, übertragen wird also zwischen dem Erzähler, den ungebildeten potentiellen Kritikern und den gebildeten Lesern unterschieden. Die ungebildeten Kritiker werden ausgegrenzt, und der Erzähler und seine gebildeten Leser feiern die Mysterien gemeinsam unter sich. Zur Bestimmung der Leserrolle in den Noctes Atticae gilt es weiter festzuhalten, welcher Art die beiden letzteren durch den Filter der Metapher erscheinen.

Wenden wir uns zunächst dem Erzähler zu. Seine Erzählaufgabe ist verknüpft mit dem narratologischen Kriterium der Stimme ${ }^{22}$ und damit mit der Frage, wer spricht. Der narratologisch unmarkierte narrative Akt ist der, in dem ein Erzähler, sei er auktorial, personal oder ein Ich-Erzähler, vom Anfang bis zum Ende der Erzählung selbst über die Ereignisse berichtet. Der narratologisch spannendere narrative Akt aber ist der, in welchem der Erzähler auch andere Sprecher zu Wort kommen lässt. In erster Linie geschieht das über Wortwechsel der Figuren, die als Zitate gekennzeichnet sind. Stärker ausgestaltet wird die Erzählsituation ferner durch die Einführung eines Binnenerzählers, ${ }^{23}$ der nicht nur für eine kurze Aussage, sondern für eine umfangreichere Erzählung, die ana- bzw. proleptisch, als mise-en-abime oder als Novelle in die erste Erzählebene eingebettet ist, das Wort erhält. Bei der Analyse des narrativen Aktes gilt es daher als erstes, zu bestimmen, wer spricht bzw. wer Stimmführer ist.

Wie auch im Rahmen dieses Zitats zeichnet sich die Haltung des Erzählers der Noctes Atticae dadurch aus, dass er besonders häufig andere Sprecher zu Wort kommen lässt. Unter Bezug auf das von Genette ${ }^{3} 2010$ entworfene narratologische Kriterium der Stimme wäre von einer häufigen Stimmenabgabe zu sprechen. Standpunkte und Darlegungen werden historischen Persönlichkeiten in den Mund gelegt und die Ausführungen dadurch tatsächlich, wenn sich die Sprecher in einer konkret entworfenen Situation gegenüber stehen, oder - im Falle eine Zitatenreihung - in einem übertragenen Sinn dialogisiert. Der Erzähler tritt zurück und übernimmt über weite Strecken die Rolle eines Moderators, der Beiträge, seien es Zitate, direkte Reden, indirekte oder paraphrasierte Worte, verwaltet. Der Sprecher, an den der Erzähler durch das Aristophanes-Zitat die Stimme abgibt, ist der Chor. Er nennt die Chöre der Mysten, zu denen er sich dazu zählt: toĩ ท̇ der Erzähler im Vorwort Aussagen über seinen Text macht, so macht der Sprecher des Chors eine Aussage über die von ihm geleitete Zusammenkunft. Der Sprecher des Chors kann damit als Metapher für den Erzähler gelesen werden. Der Erzähler führt mit anderen Worten einen Chor der Gebildeten zusammen, indem er ihre Beiträge je nachdem harmonisch oder in einer Kontroverse vereint. ${ }^{24}$

Neben dem Erzähler und seiner schriftlichen Fixierung der Kapitel umfasst die Metapher der Mysterienfeier zugleich auch die Lektüre durch den Leser. Er ist als Myste ausdrücklich

22 Zur Stimme vgl. Genette ${ }^{3} 2010,137-174$.

23 Genette ${ }^{3} 2010$, 147-152 und bes. 162 (Tabelle) spricht vom intradiegetischen Erzähler in Unterscheidung vom Autor als extradiegetischem Erzähler. Die Bezeichnung als Binnenerzähler hat demgegenüber den Vorteil, dass sie die Ebene des narrativen Aktes bereits als eine zweite Erzählebene beschreibt.

24 Vgl. Bachtin 1979, 157, der für den Roman eine Stimmenvielfalt konstatiert und die Aufgabe des Erzählers im Roman als deren Orchestrierung beschreibt: «Der Roman ist künstlerisch organisierte Redevielfalt, zuweilen Sprachvielfalt und individuelle Stimmenvielfalt. Die innere Aufspaltung der einheitlichen Nationalsprache in soziale Dialekte, Redeweisen von Gruppen, Berufsjargon ... ist die notwendige Voraussetzung für die Romangattung: der Roman orchestriert seine Themen ... mit der sozialen Redevielfalt und der auf ihrem Boden entstehenden individuellen Stimmenvielfalt.» 
und als aktiv Beteiligter in die Feier einbezogen. Das in Ran. 354 geforderte Schweigen thematisiert indirekt das Recht zur Mitsprache. Die Tatsache, dass der Myste in die kultische Handlung, etwa über eine Mahlzeit oder einen vorgegebenen Dialog, aktiv einbezogen wurde, kann als Grund für die Beliebtheit von Mysterienkulten in Rom in hellenistischer Zeit gelten. ${ }^{25}$ In den letzten anderthalb Versen wendet sich der Chor direkt an seine Mysten. Übertragen auf die Noctes Atticae richtet der Erzähler diese Worte also an die von ihm anvisierte Leserschaft, und wir erhalten Aufschluss über das Bild, das der Erzähler von ihr entwirft: Sie feiert mit dem Erzähler mit, ist in die Mysterien, d.h. in Inhalt und Form der Studien, eingeweiht und bringt ihre Stimme in den Gesang und in die Feier ein, beteiligt sich also an der Erörterung der vorgelegten Themen. Diese Involvierung schlägt sich im Text nieder durch Mittel der Stimmenabgabe, wie Zitate, indirekte Rede und Paraphrasen zu gebildeten Persönlichkeiten oder Dialoge auf der Ebene der Figuren, die, wenn sie anonym bleiben, auch als Platzhalter für den impliziten Leser dienen können. Die Reaktion des Lesers besteht dann entweder in der Identifikation ${ }^{26}$ mit dem anonymen Gesprächspartner oder in der Abgrenzung ${ }^{27}$ ihm gegenüber.

Die Leserinvolvierung kann aber auch über den fixierten Text hinausreichen, indem der implizite Leser zur Sinnstiftung zwischen Hauptthema und Anfügungen und zur Ergänzung offener Listen ${ }^{28}$ aktiviert wird. So folgt auf die Kritik an Plinius' Überlieferung von Demokrit zugeschriebenen Mirabilien in Gell. 10,12,1-8 in 10,12,9f. die Beschreibung von Favorinus' Überlieferung von einem Archytas von Tarent zugeschriebenen Mirabile. Die knappe abgrenzende Einleitung durch sed in 10,12,9 fordert den Leser zur Suche nach dem Unterschied auf, ohne dass dieser explizit gemacht würde. Der Erzähler betont durch die unkommentierte Reihung längerer und kürzerer Einheiten auch den work-in-progress-Charakter seiner commentari $2^{29}$ und lädt in dieser Weise den Leser zur Mitarbeit ein. Wenn der Erzähler, wie wir zu Praefatio 2 festhalten konnten, in erster Linie ein Leser ist, dann ist der Leser wohl zumindest auch ein Erzähler, der bei der Lektüre die Leerstellen ${ }^{30}$ zwischen den Texteinheiten ergänzt und einen - wenn nicht eine zusammenhängende Handlung erzählenden, so doch bezüglich seiner thematischen Auswahl, Ordnung und Haltung möglichst weitgehend kohärenten Text konstruiert. Eine Erklärung wie in Praefatio 2, wonach die thematische Reihung rein zufällig entstanden sei und alles unterschiedslos und vermischt notiert wurde, so wie es dem Erzähler zuteil wurde, lässt Zweifel aufkommen, ob der Text, der so sehr den Leseakt des Erzählers ins Zentrum stellt, wirklich auf einen Leser ausgerichtet ist; gewiss nicht auf einen passiv rezipierenden, sondern wohl auf einen aktiv konstruierenden.

Kaster 1988 stellt Praefatio 20f. in den Kontext zeitgenössischer Inschriften und hält fest, dass in CIL 6,16843 der Unterricht, den ein verstorbener Grammatiker genoss, als i $\varepsilon \rho \grave{n} . .$.

\footnotetext{
${ }^{25}$ Im öffentlichen Staatskult hatte der Einzelne dagegen nur die Möglichkeit, das Opferritual als Zuschauer $\mathrm{zu}$ verfolgen.

26 Vgl. u.a. Gell. 13,15; 13,29; 15,9.

27 Vgl. u.a. Gell. 13,31; 15,2; 15,30.

28 Gell. 10,25. Die Rahmenhandlung schildert, wie sich der Ich-Erzähler in einem Reisewagen sitzend die Zeit damit vertreibt, alle ihm bekannten lateinischen Begriffe für Wurfgeschosse und Wasserfahrzeuge aufzuzählen. Er kommt auf 26 bzw. 29 Begriffe und bietet damit eine gute Vorgabe. Der Ball, diese Vorgabe noch zu überbieten, liegt am Ende des Kapitels aufgrund des offenen Schlusses beim Leser. Erzähler und Leser treten hier in ein typisch sophistisches Konkurrenzverhältnis.

29 Zum Begriff vgl. Praefatio 3 u.a. Der Erzähler hat damit ein klares Bild von der Gattung seines Textes.

30 Nach Iser ${ }^{3} 1994$.
} 
$\delta$ Ło $\alpha \sigma k \alpha \lambda i ́ n$ bezeichnet wird. ${ }^{31}$ Inschriften wie diese und Stellen wie bei Chorikios, Proc.

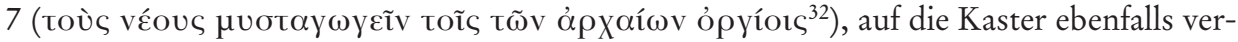
weist, zeugen von der Vorstellung, dass Bildung eine Weihe vermittelt, die nur einzelnen Auserwählten zuteil wird, von dauerhafter Wirkung ist und in ihren sozialen Konsequenzen nicht hinterfragt wird. In Vervollständigung des Bildes, das durch Ran. 354-356 und 369-371 vom impliziten Leser entworfen wird, ist festzuhalten, dass die in den Noctes Atticae vermittelte Bildung nicht allen zugänglich ist, sich nur in ihrer Anwendung offenbart - die Weihe vollzieht sich in einem performativen Akt, und der implizite Leser muss aktiv sein - und sozialen Nutzen bringt, indem sie den Zutritt zu einem Zirkel des Wissens ermöglicht.

Die Lebenszeit, die ihm bleibt, wird der Erzähler nutzen, um seine Studien fortzuführen. Er verweist damit auf den offenen Charakter seines literarischen Werks. So heisst es in Praefatio 23:

quantumque a tuenda re familiari procurandoque cultu liberorum meorum dabitur otium, ea omnia subsiciva et subsecundaria tempora ad colligendas huiuscemodi memoriarum delectatiunculas conferam.

Wieviel Musse von der Sorge um das Vermögen und die Erziehung meiner Kinder mir auch immer gegeben werden wird, diese ganze übrige und zweitrangige Zeit will ich zum Zusammentragen von Vergnüglichkeiten von Erinnerungen dieser Art aufwenden.

Das Vorwort wird durch den Hinweis auf eine Inhaltsübersicht (capita rerum ... exposuimus) abgeschlossen: Diese bringe dem Leser Nutzen. Er könne, so heisst es in Praefatio 25, daraus ersehen, wo er finden kann, was er sucht: quid quo in libro quaeri invenirique possit. Mit diesem Ziel fasst der Erzähler einen Leser ins Auge, der aufgrund seiner Amtspflichten zu beschäftigt für eine durchgehende Lektüre ist und sich ausserdem soweit für die Bildungsinhalte interessiert und in sie involviert ist, dass er mit einer vorgefassten Fragestellung an die Noctes Atticae herantritt. Wenn auch das zum Inhaltsverzeichnis angegebene Ziel als solches gerechtfertigt scheint, so stellt sich dennoch die Frage, wie gross der Nutzen für den Leser bei rund 400 Themen, die thematisch ungeordnet aufgelistet sind, tatsächlich sein kann, zumal manche Titel dieses Inhaltsverzeichnisses mehrzeilige Sätze umfassen. ${ }^{33}$ Die Inhaltsübersicht mutet eher wie eine Zusammenfassung der Sammlung an. Der Erzähler würde dann in erster Linie zeigen, dass er die gesamte Literatur noch weiter komprimieren kann. Auch diese Reduktion zeigt eine Ausgrenzungstendenz des Erzählers, der eine Welt konstruiert, die sich nur aus Texten, die er gelten lässt, zusammensetzt. ${ }^{34}$ Die Ausgrenzung anderer verweist zugleich auf die eigene Zuordnung.

\footnotetext{
31 Kaster 1988, $15 \mathrm{f}$.

32 So wird der durch den Sophisten vermittelte Unterricht beschrieben.

33 Vgl. die Überschrift zu 19,1: Ex quinque corporis sensibus duos esse cum beluis maxime communes; quodque turpis et improba est voluptas, quae ex auditu, visu odoratuque procedit, quae vero ex gustu tactuque est, rerum omnium foedissima est, cum hae duae bestiarum etiam sint, reliquiae hominum tantum.

34 Und insofern ist der Erzähler der Noctes Atticae natürlich nicht einfach nur «schwach〉. Das im Titel gewählte Adjektiv dient vielmehr mittelbar der Beschreibung der Leserrolle.
} 


\section{Kontextualisierung als Selbststilisierung: Gellius in Auseinandersetzung mit Plinius}

Die Praefatio gibt dem Leser bereits eine Vorstellung von dieser gültigen Textwelt. Mit den Zitaten von Heraklit von Ephesos, Aristophanes und dem alten lateinischen Sprichwort wird der Diskurs der folgenden Kapitel entworfen. Der Erzähler konstruiert ferner über die Titelbeispiele intertextuelle Bezüge zu anderen Werken der Miszellanliteratur und legitimiert damit sein eigenes Schaffen, indem er sich der Gruppe ihrer Autoren zuordnet. Auch innerhalb dieser Gruppe wird der Erzähler Zuordnungen und Abgrenzungen vornehmen. Riggsby 2007 nennt neben Gell. andere Miszellanschriften mit Inhaltsübersicht: Scribonius Largus, compositiones, Plinius maior, naturalis historia ${ }^{35}$ und Columella, de re rustica. Die Schaffensperiode dieser drei Autoren fällt wie diejenige des Gellius in die Kaiserzeit. Diese genannten und weitere Referenzwerke wie die Anekdotensammlung des Valerius Maximus oder Suetons Biographien bündeln das für die Mitglieder der Oberschicht relevante Wissen, wenn es darum geht, in der Deklamation oder der konvivialen Konversation zu bestehen. Sie sind gewiss als Reaktion auf die wachsende Zahl von Büchern und Ausdruck eines Bedürfnisses nach Wissensstrukturierung zu sehen. Dabei gilt es aber auch festzuhalten, dass nicht nur die Zahl der Bücher zunahm, ${ }^{36}$ sondern dass diese Bücher auch aufgrund kaiserlicher Bibliotheksstiftungen leichter zugänglich waren und mithin Unkenntnis derselben weniger leicht entschuldigt werden konnte. Die Referenzwerke boten ihren Lesern bzw. Benutzern nicht nur Zusammenfassungen einzelner Werke, sondern Bibliotheken im Taschenformat. Gellius inszeniert die Bibliothek als Schauplatz in den Kapiteln 11,17; 13,20 und 19,5.

Neben dem Inhaltsverzeichnis scheint das Vorwort einen festen Platz in der Miszellanliteratur zu haben. Vorworte, die vergleichbar sind mit demjenigen in den Noctes Atticae finden sich bei Plutarch, quaestiones convivales, Columella, de re rustica, Seneca, naturales quaestiones, Athenaios, Deipnosophistai und wiederum bei Plinius maior, naturalis historia. Beim Vergleich dieser Vorworte mit der Praefatio der Noctes Atticae sticht besonders die intertextuelle Verbindung zu Plinius ins Auge. Bei Plinius finden wir im Vorwort im Wesentlichen die gleichen Punkte in einer vergleichbaren Reihenfolge wie im Vorwort von Gellius behandelt. Die Übereinstimmung ist so gross, dass sie nicht zufällig, sondern beabsichtigt scheint. Daher soll im Folgenden auch das Vorwort von der naturalis historia zusammenfassend beschrieben werden.

Plinius eröffnet in nat. 1-11 das Vorwort mit einer Widmung an den Kaiser. Daran schliessen sich ein Lob auf die Bildung des Kaisers an und Überlegungen, was eine Widmung an den Kaiser für den Autor bedeutet. In nat. 14f. wendet sich auch Plinius gegen das Schulmässige: Das allzu Bekannte bereitet Überdruss. Er betrachtet sich dagegen als Pionier. Denn sein Thema ist das Leben selbst: rerum natura, hoc est vita, narratur. Seine Lektüreerfahrung bringt Plinius vor allem ins Spiel, um in nat. 17 durch den Umfang der gelesenen Literatur seine Kompetenz zu belegen:

\footnotetext{
35 Plinius selbst nennt Valerius Soranus als Vorgänger.

${ }^{36}$ Holford-Strevens 2003, $28 \mathrm{f}$.
} 


\begin{abstract}
XX (milia) rerum dignarum cura - quoniam, ut ait Domitius Piso, thesauros opportet esse, non libros - lectione voluminum circiter II (milia), quorum pauca admodum studiosi attingunt propter secretum materiae, ex exquisitis auctoribus centum inclusimus XXXVI voluminibus.
\end{abstract}

20000 der Beschäftigung werte Gegenstände - weswegen, wie Domitius Piso sagt, es Schätze, nicht Bücher sein müssten - haben wir durch die Lektüre von rund 2000 Bänden, die nur ziemlich wenige Forschende wegen der Entlegenheit des Stoffes in die Hand nehmen, aus 100 ausgesuchten Autoren in 36 Bänden verzeichnet.

Plinius bezeichnet seine Sammlung indirekt (über die Einschätzung eines Freundes) als Schatz. Gellius versteht die seine als Vorrat und spricht ihr damit bei vordergründiger Bescheidenheit einen höheren Wert zu. Beide Autoren konstruieren ihren Text und das Bild des Erzählers durch ein Abgrenzungs- und Ausschlussverfahren. Der Leser erhält ein Bild von der Welt der Natur bzw. von der Welt der Literatur, das erst den Auswahlkriterien des Erzählers standhalten musste.

Auch Plinius' Sammlung ist eine Frucht seiner Nachtarbeit. Er betont in nat. 18 f., dass sein Studium ihn auf diese Weise, indem er die Nacht zum Tag machte, nicht von seinen Pflichten gegenüber dem Kaiser abgehalten hat: dies vobis impendimus. Er nennt einen weiteren, privaten und buchhalterisch anmutenden Grund: Was man an Schlaf der Nacht abzieht, das lebt man mehr.

In nat. 24-26 äussert sich auch Plinius zur Titelwahl. Es fällt auf, dass einzelne der ge-

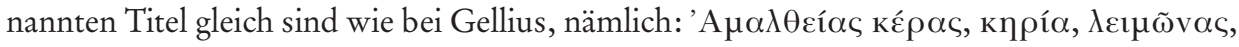

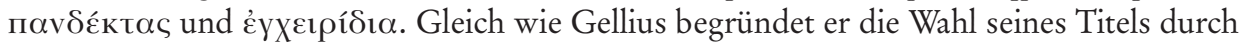
seine Bescheidenheit. Es verdriesst ihn nicht, keinen ausgefalleneren Titel gewählt zu haben: Me non paenitet nullum festiviorem excogitasse titulum. Im Gegenteil, Plinius mockiert sich über die ausgefallenen Titel vorab der griechischen Werke: ut vel lactis gallinacei sperare possis in volumine haustum. Unter den lateinischen Titeln wendet er sich gegen denjenigen eines Bibaculus, der seiner Miszellanschrift den Titel Lucubrationum gegeben habe: puto quia Bibaculus erat et vocabatur. Der Vergleich mit dem Vorwort bei Gellius zeigt, dass Gellius selbst seine Sammlung genau als lucubratiunculae bezeichnet hat.

An etwas früherer Stelle als Gellius, in nat. 27, äussert sich Plinius zur Unvollendbarkeit einer solchen enzyklopädischen Sammlung. In nat. 28-32 wendet sich auch er unter Heranziehung eines Zitats an seine Kritiker und lässt Cato, de militari disciplina für sich sprechen.

Auch Plinius schliesst sein Vorwort in nat. 33 mit einem Hinweis auf eine Inhaltsübersicht ab. Im Vergleich zu Gellius scheint der Nutzen leichter nachvollziehbar. Damit der Kaiser Zeit spart, so schreibt Plinius, und nicht alles durchzulesen braucht, kann er diese Zusammenstellung benutzen. ${ }^{37}$ Diese Inhaltsübersicht dürfte ihre Dienste tatsächlich einigermassen leisten, da bei Plinius die Kapitel thematisch geordnet sind.

Die folgende Darstellung soll die ähnliche Reihenfolge gleicher Themen verdeutlichen. Die Numerierung bei Plinius bezieht sich auf die Position der Punkte in seinem Vorwort:

${ }^{37}$ Zum Inhaltsverzeichnis bei Plinius hält Krasser 2005, 373-375 fest, dass Werke wie Plinius, nat. den Leser in den Vordergrund rückten. Als Wissensspeicher sei ihre Form von funktionalen Interessen bestimmt. Folglich sind die Vorworte von nat. und Gell. auch in der Rolle, die dem impliziten Leser zugeschrieben wird, vergleichbar. 
Gellius, Noctes Atticae

1. den eigenen Kindern gewidmet

2. Lektüreerfahrung: litterarum penus

3. bescheidener Titel (festivitates)

4. Nachtarbeit: Spielerei (lucubratiunculae)

5. gegen das Schulmässige

6. gegen Kritiker: Aristophanes, Ran.

7. Unvollendbarkeit (subsiciva tempora)

8. Hinweis zur Inhaltsübersicht
Plinius maior, naturalis historia

1. dem Kaiser gewidmet

3. Lektüreerfahrung: thesaurus

5. bescheidener Titel (festiviorem)

4. Nachtarbeit: Buchhaltung (subsicivis temporibus)

2. gegen das Schulmässige

7. gegen Kritiker: Cato, de militari disciplina

6. Unvollendbarkeit

8. Hinweis zur Inhaltsübersicht

Obwohl sich der Erzähler durch die Übereinstimmungen in Reihenfolge und Thematik in die Nähe zu seinem Vorgänger Plinius stellt, wendet er die Aussage immer wieder gerade gegensätzlich zu Plinius und nimmt dadurch eine Abgrenzung vor, ${ }^{38}$ ohne dass Plinius namentlich genannt würde. So sehen wir die staatliche Widmung von nat. bei Gellius ins Private gewendet. Aus dem Schatz (thesaurus) bei Plinius wird ein Vorrat (litterarum penus). Während in nat. allein der Nutzen, der die Nachtarbeit gegenüber einer bei Tage betriebenen literarischen Arbeit bringt, zählt, wertet der Erzähler in Gell. seine nächtliche Tätigkeit als Spielerei bei Kerzenschein, ohne ausdrücklich auf den Nutzen einzugehen, der sich aus der Verlegung der Spielerei in die nächtlichen Stunden ergibt. Der Erzähler bei Plinius mockiert sich ferner über die Aufgeblasenheit anderer Titel, während sich der Erzähler in Gell. vom Esprit ebendieser Titel angetan zeigt. Auffallend ist die wörtliche Entsprechung $\mathrm{zu}$ festiviorem in nat., die in den Noctes Atticae durch festivitates hergestellt wird. ${ }^{39}$ Im Kontext des gesamten intertextuellen Bezugs der Praefatio von Gellius auf diejenige von Plinius dürfte eine ironische Lesart dieser Anspielung gerechtfertigt sein. Der Titel naturalis historia verkündet feierlich Grosses. ${ }^{40}$ Durch diese wörtliche Aufnahme und die gleichzeitige Aufnahme des Titels in die Liste der Beispiele hält der Erzähler in Gell. den Finger darauf, dass <naturalis historia der von Plinius geforderten massvollen Haltung in der Titelwahl entgegensteht. Allgemein gehört Plinius zu den Quellen, die der Erzähler am harschesten tadelt. ${ }^{41}$ Ähnlich ergeht es Seneca, dessen Werk mit epistulae morales ebenfalls genannt ist und der in Gell. 12,2 schlechte Kritik vom Erzähler erhält. ${ }^{42}$ Schliesslich belegt der Erzähler in nat. seine grosse Belesenheit durch Zahlen. Der Erzähler in Gell. dagegen wendet sich gegen Vielwisserei (по $\nu \mu \alpha{ }$ ín) und betont seine sorgfältige Auswahl des Stoffes. In dieser Gegenüberstellung dürfte ebenfalls eine Aussage zu nat. und ihrem Verfasser zu sehen sein. ${ }^{43}$

\footnotetext{
38 Ähnliches halten Minarini 2000 und Faider 1927, 209 fest.

39 So auch Minarini 2000, 539.

40 Deutlich profaner wäre etwa: De rebus naturalibus.

41 Vgl. Gell. 9,4 und 10,12.

42 Nachdem der Erzähler in 12,2,1 das abwertende Urteil angeführt hat, das Seneca bezüglich Stil und Inhalt seitens der zeitgenössischen Literaturkritik erhält, begründet er seine eigene Abwertung Senecas auf dessen Kritik an Ennius, Cicero und Vergil. Die Wortwahl des Erzählers spricht eine klare Sprache: Seneca urteile geschmacklos (insulsissime in 12,2,5), sei ein Schwätzer (bomo nugator in 12,2,8), inkompetent und einfältig (inepti et insubidi bominis ioca in 12,2,11) und was ihm gelungen sei, wiege seine stilistischen und inhaltlichen Vergehen bei Weitem nicht auf (12,2,12-14). Diese invektivische Abwertung Senecas als Kritiker der archaischen und klassischen lateinischen Autoren Ennius, Cicero und Vergil ist als Ausdruck der antiquarischen Tendenz der Noctes Atticae und der zweiten Sophistik überhaupt zu verstehen. Allgemeiner nimmt der Erzähler der Noctes Atticae eine Abgrenzung gegenüber dem - nicht sozial, aber literaturhistorisch neben Plinius nächsten Vorgänger vor.

43 So auch Minarini 2000, 540.
} 
Beide äussern sich zum Umgang mit Kritikern, und beide geben dabei durch ein Zitat ihre Stimme an eine Persönlichkeit ab, doch könnte die Wahl kaum unterschiedlicher ausfallen: Cato maior wird Aristophanes gegenüber gestellt.

\subsection{Die zweite Sophistik als Bewegung des starken Lesers}

Wenn sich der Erzähler der Noctes Atticae nun innerhalb seiner allgemeinen Zuordnung zu Plinius als Miszellanautor doch wiederum von ihm distanziert, so bleibt zu bestimmen, in welche Richtung diese Distanznahme führt und auf welche Gruppe sich der Erzähler dabei zubewegt. Vieles spricht dafür, das vom Erzähler entworfene Selbstbild als Zuordnung zur zweiten Sophistik zu verstehen. Der Begriff der zweiten Sophistik bezeichnet in seiner ursprünglichen Konzeption eine kulturelle Bewegung, die genuin griechisch ist, ${ }^{44}$ insofern als sich die ausgeprägte Besinnung auf die eigene griechische Kultur aus der politischen Situation der hellenischen Welt in der hohen Kaiserzeit ableiten lässt. Die griechische Kultur übernimmt hier in einer von der römischen militärischen und politischen Überlegenheit dominierten Ordnung identitätsstiftende Funktion, indem sie das politische Vakuum durch das Bewusstsein kultureller Überlegenheit kompensiert. Trotz dieser Verankerung der zweiten Sophistik in der griechischen Lebenswelt hat die Forschung der vergangenen Jahrzehnte gezeigt, dass der römischen Kultur auch hier, getreu dem alexandrinischen Konzept von imitatio und aemulatio, eine eigene Interpretation eines griechischen Phänomens gelang. Das politische Vakuum, das die griechische Oberschicht empfindet, trifft auch die römische Nobilität. Auch ihr bieten sich als Kompensation und als neues distinktives Unterscheidungsmerkmal gegenüber der übrigen Bevölkerung Kultur und Bildung an, ${ }^{45}$ die im Hellenismus nicht mehr rein römisch sein kann. ${ }^{46}$ Schon die Briefe von Plinius dem Jüngeren zeugen vom Bestreben, die eigene Bildung und Kultur öffentlich zu belegen und sozialer Distinktion dienstbar zu machen. ${ }^{47}$ Die Stiftung kultureller Institutionen wie die in epistulae 1,8 beschriebene Einrichtung einer öffentlichen Bibliothek durch Plinius in seiner Heimatstadt Comum gewinnt in ihrer sozialen Bedeutung den Stellenwert politischer Aktivität. Der öffentliche Auftritt ist nicht mehr so sehr politisch, als vielmehr kulturell gesteuert. ${ }^{48}$ Dass Plinius die Bibliotheksstiftung als Tat versteht, die ihm öffentlichen Ruhm einträgt, geht daraus hervor, dass er gegenüber dem Adressaten von 1,8 seine Bedenken gegenüber einer Publikation seiner Einweihungsrede äussert. Diese würde nämlich seiner Bescheidenheit (modestiam nostram in 1,8,5) entgegenstehen. Auch Statius' Silvae gehören in diesen Kontext, behandeln sie doch in dichterischer Form die gleichen Themen wie Plinius in seinen Briefen und die Autoren der Miszellanliteratur. Beide, Plinius und Statius, haben ferner Titel gewählt, die sich unauffällig in die Titelliste in Praefatio 5-9 der Noctes Atticae

44 Grundlegend dazu Bowie 1970; vgl. auch Whitmarsh 2001.

45 Schmitz 1997.

46 Zum Bilingualismus vgl. Adams 2003. In den Noctes Atticae zeugen die zahlreichen griechischen Begriffe, die in der Erzählerrede verwendet werden, vom fortgeschrittenen Bilingualismus; vgl. Gell. 1,5,3 (als Zitat zu Q. Hortensius); 1,7,19; 1,22,12; 2,17,11 u.a. Bereits Cicero benutzt in den Briefen immer wieder griechische Ausdrücke, die nicht auf ein Zitat zurückgehen; vgl. Att. 2,9,4; 2,12,4; 2,15,4, ad. Q. fr. 3,3 u.a.

47 Dazu Ludolph 1997.

48 Vgl. Fantham 1996, 201. 
einreihen liessen. Die breite der eigenen Bildung lässt sich am besten durch die thematische Breite einer Miszellanschrift belegen. Das gilt - mit allen sozialen Implikationen - für den Rezipienten, der sich zum Adressatenkreis des Werkes zählt, nicht weniger als für den Verfasser. Entsprechend dieser sozialen Voraussetzungen und Entwicklungen im römischen Literaturbetrieb gelten in der Forschung seit Harrison 2000 sophistische Züge in der lateinischen Literatur besonders für Apuleius als erwiesen. So scheint es gerechtfertigt, nach Apuleius auch dessen Zeitgenossen (und möglichen Kommilitonen ${ }^{49}$ in Athen) Gellius auf Einflüsse der zweiten Sophistik hin zu untersuchen. Nachdem Holford-Strevens 2003 und Keulen 2009 erste Ansätze dazu geboten haben, sollen hier die Stellen und Aussagen aus dem Vorwort aufgeführt werden, die den Erzähler der Noctes Atticae in die Nähe der zweiten Sophistik rücken. ${ }^{50}$

Eine Annäherung von Gellius an die zweite Sophistik legt zum einen die gezielte Zuordnung und Ausgrenzung auf textlicher und sozialer Ebene nahe. Abgrenzungsstrategien gegenüber der ungebildeten Masse, dem profanum volgus in Praefatio 20, und Konkurrenz zwischen den gebildeten Rednern gehören zu den Charakteristika der Vertreter der zweiten Sophistik. ${ }^{51}$ Wir konnten bereits sehen, wie das Aristophanes-Zitat als Mittel zur Abgrenzung auf der Leser-Seite dient. Typisch ist an dieser Abgrenzung ferner die weitere Differenzierung unter den Gebildeten, bei denen zwischen dem Bildungsadel, der ein reflektiertes Verhältnis zur für ihn alltäglichen Bildung aufweist, und den «Neugebildeten〉, die das anständige Mass in der Bildung und ihrer Zurschaustellung nicht zu wahren wissen ${ }^{52}$ unterschieden wird. Auch bei Gellius ist das Bewusstsein für das richtige Mass und die richtige Form an Bildung zu erkennen, wenn er in Praefatio 12 gegen Vielwisserei Stellung nimmt. Vielwisserei thematisiert auch Apuleius in met. 9,13,4f. (anhand des Adjektivs multiscius), wo sie wie bei Gellius als eine reine Anhäufung von Erfahrungen, die ohne Einfluss auf den Charakter und die Lebenshaltung bleiben, abgelehnt wird.

Ergänzt wird diese Zuordnung zur zweiten Sophistik durch die Verbindung von Bildungsinhalten und dem Anspruch moralischer Charakterformung einerseits und Unterhaltung, Spiel und Witz andererseits, die der Erzähler der Miszellanschrift und ihrem Entstehen verleiht. Diese für die zweite Sophistik charakteristische Verbindung wird heute in der Literatur gern mit dem Begriff des spoudaiogeloion beschrieben. ${ }^{53}$ Zimmerman 2008 erläutert ihn

${ }^{49}$ Dazu Holford-Strevens 2003, 22-26. Von einer persönlichen Bekanntschaft zwischen Apuleius und Gellius auszugehen, scheint am ehesten gerechtfertigt aufgrund der Annahme, dass der in Gell. 19,11,3 genannte amicus meus, dessen Nachdichtung eines Platon zugeschriebenen Epigramms Gellius zitiert, mit Apuleius zu identifizieren ist. Apuleius verteidigt seine Epigramme in apol. 10,7, indem er Platon als Vorbild nennt: etiamne, Aemiliane, si Platonis ipsius exemplo doceo factos. Doch muss die Diskussion bis heute spekulativ bleiben.

${ }^{50} \mathrm{Zu}$ Gellius als Vertreter der zweiten Sophistik vgl. Keulen 2009, 2: «... the time seems ripe for a project to integrate Gellius and his readers in their cultural, social and political context, ... the Greek cultural phenomena also known as the «Second Sophistic`.» Holford-Strevens 2003 integriert die Kontextualisierung an prominenter Stelle in den Titel: «Aulus Gellius. An Antonine Scholar and his Achievement».

51 Als ein Beispiel für viele mag Apuleius, apol. stehen. Hier versucht der Angeklagte wiederholt, am ausgeprägtesten aber im Proömium und in der Peroratio, sich gegenüber den nach seiner Beschreibung ungebildeten Anklägern abzugrenzen und dagegen mit dem Richter, per se ein Vertreter der gebildeten Oberschicht, zu verbünden. Auch die für die zweite Sophistik typische Parodie ist in ihrer unmittelbaren Gegenüberstellung zur Vorlage ein Konkurrenzprodukt.

52 Schmitz 1997.

${ }^{53} \mathrm{Zu}$ diesem Konzept gehört auch das Gelächter des Gebildeten, worüber Riess 2008, xiii schreibt: «Through this integrative concept of the erudite laugh, literature and life merge into an inseparable unity.» Wie sich 
als Eigenschaft von Apuleius' met. und führt das in ihm zum Ausdruck kommende Kon-

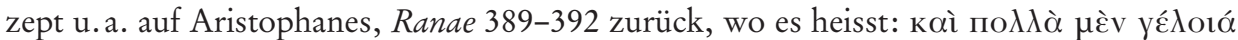

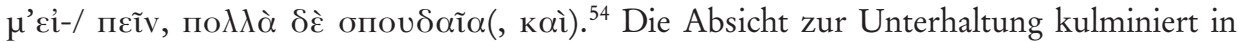
met. 1,1 in der direkten Wendung an den Adressaten: lector intende, laetaberis. Daneben ist den einzelnen Erzählungen, die folgen, aufgrund ihrer satirischen Züge auch eine moralisierende Haltung inhärent. Was bei Apuleius zugespitzt wird und auch durch den HypsosBathos-Wechsel der Epos-Parodie ${ }^{55}$ in met. zum Ausdruck kommt, ist bei Gellius ebenfalls zu erkennen. ${ }^{56}$ Denn neben der Bezeichnung als lucubratiunculae für die nächtliche Exzerpiertätigkeit des Bildungshungrigen steht das Wort ludere in Praefatio 4: commentationes hasce ludere ac facere exorsi sumus. Das Ergebnis dieser demnach spielerischen Tätigkeit sind zwar Vergnüglichkeiten, ad colligendas huiuscemodi memoriarum delectatiunculas in Praefatio 23., aber doch solche, die der Bildung dienen, wie der Erzähler in Praefatio 12 erklärt: eaque sola accepi, quae aut ingenia prompta expeditaque ad honestae eruditionis cupidinem ... ducerent. Rein enzyklopädisches Wissen dagegen wird in Gell. 14,6 rundwegs und in den zahlreichen Grammatikersatiren indirekt abgelehnt. Auf der anderen Seite ist auch der Leser in Praefatio 14 als einer zu denken, der sich aus Vergnügen (voluptas) an die Lektüre macht. Unterhaltung und Bildung sind bei Gellius als eins gedacht. Plinius dagegen betont vielmehr den Nutzen seiner Tätigkeit. Neben dem beschriebenen Nutzen der Nachtarbeit fordert er in nat. 16 (gegen Livius) auch einen Nutzen des in dieser Nachtarbeit entstandenen Textes: maius meritum esset operis amore, non animi causa, perseverasse et hoc populo Romano praestitisse, non sibi. Spiel und Unterhaltung haben in Plinius' Vorwort keinen Platz.

Zwar werden, so hält der Erzähler in Praefatio 13 fest, anspruchsvolle Themen aus verschiedenen Fachgebieten erläutert, doch betont er dabei, dass die Fragen nicht in ihrer Tiefe behandelt, sondern nur als Kostproben (libamenta) angetippt würden. So bleibt etwa die technische Beschreibung der fliegenden Holztaube, die Favorinus in Gell. 10,12,9f. als Erfindung Archytas von Tarent zuweist, denkbar vage: ratione quadam disciplinaque mechanica. Gell. 14,2 schildert den Ich-Erzähler, der sich als Richter mit dem moralisch kontroversen Fall konfrontiert sieht, in dem ein Mann mit gutem Leumund von einem Mann mit schlechtem Leumund die Rückerstattung eines Darlehens fordert, ohne den Beweis erbringen zu können, je ein solches Darlehen gewährt zu haben. Auch wenn sich der IchErzähler für das moralische Dilemma so sehr interessiert, dass er den Fall mit dem Philosophen Favorinus diskutiert, ist es ihm nicht möglich, den Fall zu einer befriedigenden Lösung zu führen. Das Kapitel endet damit, dass der Erzähler den Fall abgibt, und dies, obwohl Favorinus ihm durchaus einen pragmatischen Rat erteilt hat. Auch diesen Kostproben-Charakter, dem die Erörterungen in den einzelnen Kapiteln durchwegs treu bleiben, teilen die Noctes Atticae mit anderen Texten der zweiten Sophistik. Insbesondere kann auf

auch bei Gellius Literatur und Leben überlagern, wird in den narrativen Kapiteleinleitungen deutlich, die Erlebnisse des Ich-Erzählers, wie beispielsweise seinen Besuch bei einem Buchhändler in Brundisium in 9,4 oder seine Begegnung mit dem Stoiker in 19,1, der aus seinem Reisegepäck prompt ein Büchlein mit dem passenden Zitat zücken kann, schildern. In ihnen wird eine Selbststilisierung des Ich-Erzählers nach literarischen Mustern fassbar. So ist der Krankenbesuch einerseits Teil der Rahmenhandlung in Gell. 2,26; 7,9; 12,5; 18,10 und 19,10 und andererseits narrativer Kontext innerhalb der Anekdote von Gell. 13,2.

54 Zimmerman 2008, 136-138.

55 Harrison 2000, 223.

56 Zur Unterhaltung und Belehrung bei Apuleius und Gellius allgemeiner auch Steinmetz 1982, $239-295$. 
die zeitgenössischen Romane von Achilleus Tatios sowie Philostratos und ihre enzyklopädischen Exkurse hingewiesen werden..$^{57}$

Schliesslich rückt auch die zentrale Bedeutung des korrekten sprachlichen Ausdrucks und des gepflegten Stils in der Praefatio den Erzähler in die Nähe der zweiten Sophistik. Er erhofft sich in Praefatio 16 aus seinen Beiträgen für den Leser eine kunstfertigere Rede, einen korrekteren sprachlichen Ausdruck sowie ein vornehmeres Vergnügen in der Musse und in der Unterhaltung, mithin eine angemessenere Freizeitgestaltung: oratio sollertior aut sermo incorruptior aut delectatio in otio atque in ludo liberalior. Davon, wie zentral der sprachliche Ausdruck im 2. Jh. n. Chr. im griechischen Raum war, um die Zugehörigkeit zur Oberschicht zu demonstrieren, zeugen Werke wie Lukians Lexiphanes oder die Stilisierung in Athen. 9,401d-e, wonach der Gast Ulpian sich weigerte, von Speisen zu essen, deren Name attisch nicht belegt war. ${ }^{58}$

Neben den erläuterten Erscheinungen der zweiten Sophistik wie Strategien sozialer Abgrenzung, der Überlagerung von Witz und Bildung, enzyklopädischer Wissenspräsentation (statt fachspezifischer Analyse) in Verbindung mit unterhaltender Erzählung sowie der sozial distinktiven Funktion von Sprache und Stil ist auch die Bedeutung der Performanz in der Kultur der Schaureden zu nennen. Diesem letztgenannten Punkt scheinen die Noctes Atticae als Lesetext und Produkt eines sich bibliophil ${ }^{59}$ gebenden Erzählers auf den ersten Blick sicher entgegen zu stehen. Doch führt uns das Thema der Performanz geradewegs an den Anfang unserer Darstellung des Vorwortes der Noctes Atticae und damit zurück zu den Mitteln der Leserinvolvierung. Der starke Leser vermag, so konnte gezeigt werden, den Text mitzugestalten. Sein Lektüre-Akt ist der performative Akt einer Feier, die er gemeinsam mit dem Erzähler und anderen starken Lesern begeht. Jeder dieser Leser führt die vorgelegten Kostproben zu Ende, in die vorgegebene und vielleicht auch in eine andere Richtung, gerade so wie jeder Hörer mit dem Redner interagieren, ihn seiner Aufmerksamkeit versichern und dessen Rede stellenweise fortsetzen kann. Baumann 2011 beschreibt ähnlich, wie die thematische Variation von Philostrats Eikones rezeptionsästhetisch in Leerstellen resultiert, die den Leser zur Hypothesenbildung über den Fortgang des Textes aktivieren und mit Blick auf den Erzähler die Illusion einer Performanz eines Sprechers hervorrufen. ${ }^{60} \mathrm{Ko}-$ renjak 2000 erörtert die auch indirekt über ihren Tadel zu erschliessende rhetorische Praxis der zweiten Sophistik, den Hörern über eine Rhythmisierung der Sätze, über Parallelismen und Homoioteleuta sowie Zitate aus klassischer Prosa und Dichtung die Vorwegnahme der Schlüsse zu ermöglichen. ${ }^{61}$ Der Hörer, der sich zur Gemeinschaft der Gebildeten zählen darf, ist damit ein starker Hörer, der sich in die Gestaltung der Rede einzubringen vermag.

Der Erzähler verfolgt mit seinem Text eine wahrnehmbare Wirkung auf den Leser, indem dieser auf unterhaltsame Weise sprachlich, literarisch und sozial kompetenter wird. Es konnte gezeigt werden, dass die Leserinvolvierung in der Textstruktur angelegt ist, und

${ }^{57}$ Rommel 1923 hält fest, dass Achilleus Tatios besonders Ausführungen über die Liebe und als solche interpretierbare Erscheinungen bei Tieren, Pflanzen und Steinen einfügt. Die enzyklopädischen Ausführungen stehen damit in einer inhaltlichen Beziehung zum narrativen Hauptteil.

${ }^{58} \mathrm{Vgl}$. Schmitz 1997, 75 zur Stelle.

59 Bspw. Gell. 9,4.

${ }^{60}$ Baumann 2011, 117-164.

${ }^{61}$ Korenjak 2000, 132-136, unter Verweis auf Beispiele. Ganz passend zur Mysterienfeier in Aristophanes, Ran. vergleicht Korenjak 2000, 136, diese Praxis mit dem Einbezug der Gläubigen in der katholischen Liturgie. 
zwar nach dem Konzept der zweiten Sophistik. Mittel dazu sind solch literarische Gestaltungsmuster wie der intertextuelle Bezug auf das Vorwort von Plinius, die Aktivierung des Lesers, der offene Listen und halbe Informationen ergänzt, die dem Leser überlassene Sinnstiftung durch die Konstruktion von Verbindungen über disparate Texteinheiten hinaus, die Vorstellung vom Leser als Benutzer des Textes, auf der das Inhaltsverzeichnis gründet, sowie die Engführung von Myste und Leser. Die Exklusivität des Lesers und seine Rolle als Mitwissender sind eng verknüpft mit seinem Status als aktiver Leser. Je aktiver die Rolle des Lesers ist, umso entscheidender ist für die Deutung und Rekonstruktion des Textes, wer den Text liest. Davon zeugt das Spiel von Zuordnung und Ausgrenzung, das die Praefatio der Noctes Atticae für den ganzen Text vorzeichnet und das Genette auch für den narrativen Akt in $A$ la recherche du temps perdu und den modernen Roman überhaupt herausarbeitet:

Der wahre Autor der Erzählung ist nicht nur der, der sie erzählt, sondern auch, und mitunter noch mehr, der, der sie hört. Und das ist nicht unbedingt der, an den man sich wendet: es gibt immer Leute nebenan. ${ }^{62}$

\section{Literaturverzeichnis}

Adams, J. N., Bilingualism and the Latin Language, Cambridge 2003.

Ameling, W., Aulus Gellius in Athen, Hermes 112, 1984, 484-490.

Bachtin, M., Die Ästhetik des Wortes, Frankfurt am Main 1979.

Baumann, M., Bilder schreiben, Virtuose Ekphrasis in Philostrats «Eikones`, Berlin / New York 2011.

Beall, St. M., Civilis eruditio. Style and content in the ‘Attic nights` of Aulus Gellius, Berkeley 1988.

Ders., Aulus Gellius 17,8: Composition and the Gentleman Scholar, CPh 94, 1999, 55-64.

BlAck, M., Metaphor, in: PAS 55, 1954, 273-294; wieder abgedruckt als: Die Metapher, in:

A. Haverkamp (Hg.), Theorie der Metapher, übers. von M. Smuda, Darmstadt ${ }^{2} 1996,55-79$.

Bowie, E., Greeks and their past in the Second Sophistic, P\&P 46, 1970, 3-41.

Cavazza, F., Aulo Gellio, Le notti Attiche, vol. 1, Bologna 1985.

Dover, K., Aristophanes, Frogs, Oxford 2002.

Engert, M., Gellius als Quelle bekannter und unbekannter Autoren - Zur Funktion der Tauros-Figur in der Darstellung der Lehre Platons in den Noctes Atticae, WJA 35, 2011, 123-145.

Faider, P., Auli Gellii noctium Atticarum Praefatio, Le Musée Belge 31, 1927, 189-216.

Fantham, E., Roman Literary Culture. From Cicero to Apuleius, Baltimore / London 1996.

Genette, G., Die Erzählung, übers. von Andreas Knop, Paderborn ${ }^{3} 2010$.

Harrison, St., Apuleius. A Latin Sophist, Oxford 2000.

Holford-Strevens, L., Aulus Gellius. An Antonine Scholar and his achievement, Oxford 2003.

Iser, W., Der Akt des Lesens. Theorie ästhetischer Wirkung, München ${ }^{3} 1994$.

Kaster, R. A., Guardians of language: the grammarian and society in late antiquity, Berkeley/ Los Angeles/ London 1988.

Ker, J., Nocturnal Writers in Imperial Rome: The Culture of Lucubratio, CPh 99, 2004, 209-242.

${ }^{62}$ Genette ${ }^{3} 2010,170$. 
Keulen, W., Gellius the Satirist. Roman Cultural Authority in Attic Nights, Leiden / Boston 2009.

Korenjak, M., Le Noctes Atticae di Gellio: i misteri della paideia, SIFC 16, 1998, 80-82.

Ders., Redner und Publikum: Ihre Interaktion in der sophistischen Rhetorik der Kaiserzeit, München 2000.

Krasser, H., Universalisierung und Identitätskonstruktion. Formen und Funktionen der Wissenskodifikation im kaiserzeitlichen Rom, in: G. Oesterle (Hg.), Erinnerung, Gedächtnis, Wissen: Studien zur kulturwissenschaftlichen Gedächtnisforschung, Göttingen 2005, 357-375.

Ludolph, M., Epistolographie und Selbstdarstellung, Tübingen 1997.

Marache, R., La préface d'Aulu Gelle. Couples et séries de synonymes ou de mots analogues, in: M. Simonetti et al. (Hgg.), Letterature comparate. Problemi e metodo. Studi in onore di Ettore Paratore, vol. 2, Bologna 1981, 785-791.

Marshall, P. K., Gellii Noctes Atticae, Oxford 1968.

Minarini, A., La Prefazione delle Noctes Atticae: Gellio fra Plinio e Seneca, Bollettino di studi latini 30, 2000, 536-553.

Pausch, D., Livius und der Leser. Narrative Strukturen in ab urbe condita, München 2011.

Riess, W. (Hg.), Paideia at play: learning and wit in Apuleius, Groningen 2008.

Riggsby, A. M., Guides to the wor(l)d, in: J. König and T. Whitmarsh (Hgg.), Ordering knowledge in the Roman empire, Cambridge / New York 2007, 88-107.

Rommel, H., Die naturwissenschaftlich-paradoxographischen Exkurse bei Philostratos, Heliodoros und Achilleus Tatios, Stuttgart 1923.

Schmitz, T., Bildung und Macht: Zur sozialen und politischen Funktion der zweiten Sophistik in der griechischen Welt der Kaiserzeit, München 1997.

Steinmetz, P., Untersuchungen zur römischen Literatur des zweiten Jahrhunderts nach Christi Geburt, Wiesbaden 1982.

Vardi, A., Why Attic Nights? Or: What's in a name?, CQ 43, 1993, 298-301.

Whitmansh, T., Greek Literature and the Roman Empire. The Politics of Imitation, Oxford 2001.

Zimmerman, M., Cenatus solis fabulis? A Symposiastic Reading of Apuleius' Novel, in: W. Riess (Hg.), Paideia at play: learning and wit in Apuleius, Groningen 2008, 135-155. 